\title{
00000 Sideway Aspiration Technique for Technical Thrombus in a Sharply Bifurcated Note Branch of a Large Vessel
}

Masaoki Hidaka, ${ }^{1}$ Takeshi Uwatoko, ${ }^{1}$ Taichiro Mizokami, ${ }^{2}$ Hideki Iwashita, ${ }^{2}$ Eiji Higashi, ${ }^{1}$ Keisuke Ido, ${ }^{2}$ Kenichi Matsumoto, ${ }^{2}$ and Hiroshi Sugimori ${ }^{1}$

Objective: Standard thrombectomy techniques, including stent retrieval and a direct aspiration first pass technique, are not effective when the occluded vessel is narrow and sharply bifurcated from the main trunk. Here, we present a new and alternative method for patients with such anatomical features and describe two cases treated by this method.

Case Presentations: Case 1 was a 66-year-old woman who presented with consciousness disturbance and left hemiparesis. MRA suggested a right middle cerebral artery occlusion. Case 2 was an 86-year-old man who presented with sudden onset of consciousness disturbance; MRA indicated occlusion of the basilar artery. Both cases were successfully treated by our new method. First, we navigated a microcatheter, microguidewire, and aspiration catheter into the patent and visible branch adjacent to the occluded branch. The aspiration catheter was then carefully pulled back with continuous mechanical aspiration. When reverse blood flow from the aspiration catheter was suspended, we slightly advanced the catheter and caught the thrombus. Complete recanalization was attained in the two cases treated with this technique, which was named as sideway aspiration technique (SAT).

Conclusion: Although it warrants further study, SAT may be a potentially safe and effective method for thrombectomy in cases with thrombus in a sharply bifurcated branch.

Keywords > a direct aspiration first pass technique, ischemic stroke, technical note, thrombectomy

\section{Introduction}

Recent randomized controlled trials have shown improved outcomes of acute ischemic stroke by mechanical thrombectomy. Standard techniques such as the stent retriever technique, a direct aspiration first pass technique (ADAPT), and their combined use have been reported to achieve good recanalization rates. ${ }^{1,2)}$ However, occasional cases are encountered wherein occluded branches are sharply

${ }^{1}$ Department of Cerebrovascular Medicine, Stroke Center, Sagaken Medical Centre Koseikan, Saga, Saga, Japan

${ }^{2}$ Department of Neurosurgery, Stroke Center, Saga-ken Medical Centre Koseikan, Saga, Saga, Japan

Received: June 2, 2021; Accepted: July 5, 2021

Corresponding author: Masaoki Hidaka. Department of Cerebrovascular Medicine, Stroke Center, Saga-ken Medical Centre Koseikan, 400, Kasemachi Nakabaru, Saga, Saga 840-8571, Japan Email: MasaokiHidaka@gmail.com

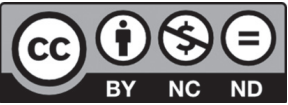

This work is licensed under a Creative Commons Attribution-NonCommercialNoDerivatives International License.

(C)2021 The Japanese Society for Neuroendovascular Therapy bifurcated from the main arteries. When introducing a microguidewire or microcatheter into the invisible occluded artery, there is a certain risk of vessel perforation.

In such cases, we considered using a novel method wherein we inserted an aspiration catheter into a remaining patent and visible branch. Then, we pulled back the catheter and caught the thrombus in the occluded branch from the side during catheter drawing. This technique was named as sideway aspiration technique (SAT). Here, we present two cases with occluded branched arteries treated with SAT to achieve complete recanalization safely.

In case of catheterization failure due to vascular tortuosity, we tried the following technique (Fig. 1). Initially, we navigated a microcatheter into the patent and visible branch that was distal to a branch point of an occluded branch over a microguidewire. Then, we deployed an aspiration catheter that was connected to an aspiration pump coaxially along with a microcatheter and microguidewire (Fig. 1A). After the aspiration catheter was delivered to the visible branch, the microguidewire and microcatheter were both drawn out and the aspiration catheter was carefully pulled back (Fig. 1B). During the procedure, we continuously 

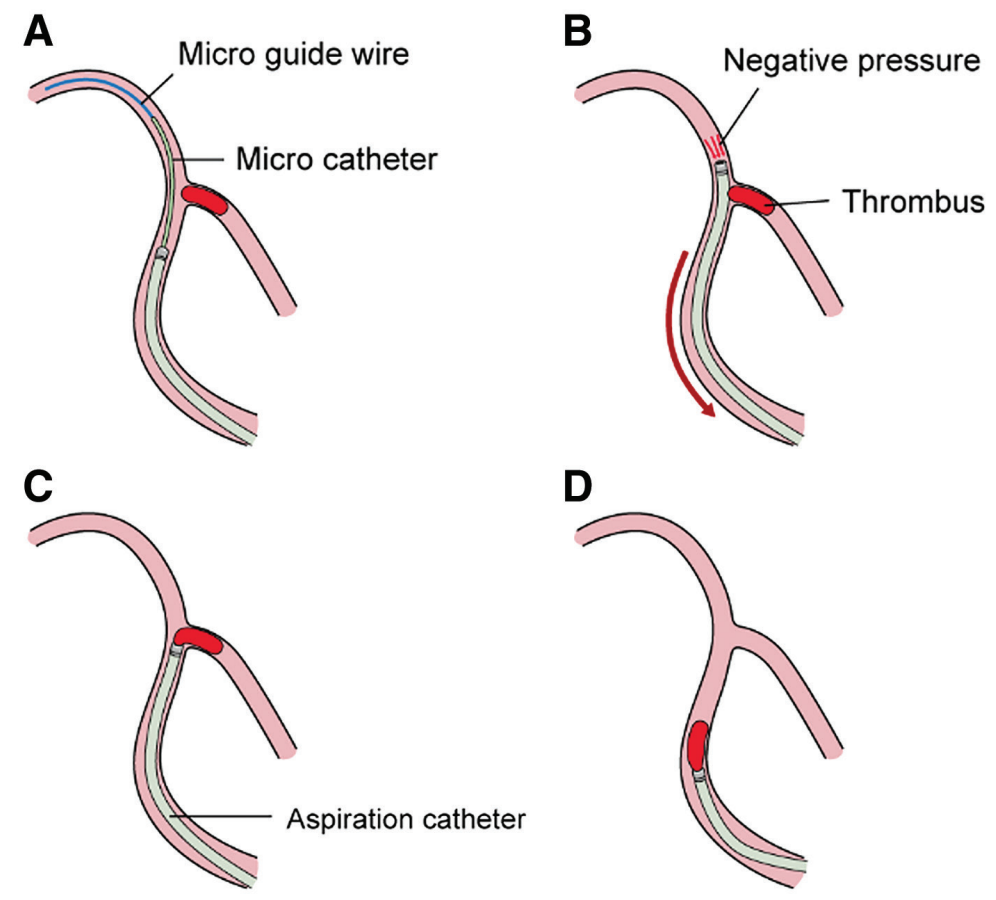

Fig. 1 An illustration of the SAT. (A) A microcatheter is advanced into the patent artery over a microguidewire. An aspiration catheter is deployed over the microcatheter and microguidewire. (B) After the microguidewire and microcatheter are both extracted, the aspiration catheter is carefully pulled back with continuous mechanical aspiration. (C) When the reverse flow is suspended, we slightly push the aspiration catheter forward to engage the thrombus. (D) After waiting for a while, the aspiration catheter is pulled out in the same way as in ADAPT. ADAPT: a direct aspiration first pass technique; SAT: sideway aspiration technique

applied negative pressure to the catheter and monitored the reverse blood flow in the pump. When the reverse blood flow markedly decreased, we expected the head of the aspiration catheter to be positioned at the proximal end of the thrombus (Fig. 1C). Thereafter, we slightly pushed forward the aspiration catheter to catch the thrombus. After approximately 30 seconds, we pulled out the aspiration catheter in the same way as in ADAPT (Fig. 1D).

\section{Case Presentations}

\section{Case 1}

A 66-year-old woman with consciousness disturbance and left hemiparesis was presented to our hospital. MRI showed faintly high-intensity areas in the cortices of the right frontal lobe, parietal lobe, temporal lobe, and insula on diffusion-weighted images (DWI). Right middle cerebral artery (MCA) occlusion was suspected on MRA.

Under local anesthesia, a 9 Fr Optimo balloon guide catheter (Tokai Medical Products, Aichi, Japan) was placed in the right internal carotid artery with systemic heparinization. Cerebral angiography revealed occlusion at the M2 segment of the right MCA. We reviewed the initial head CT scan and identified that the patient's MCA was trifurcated. ${ }^{3)}$ One of the M2 branches was totally occluded and the other two branches were patent and visible (Fig. 2A). We attempted to navigate a Marksman (Medtronic, Minneapolis, MN, USA) into the occluded branch coaxially with a CHIKAI 14 (Asahi Intecc, Aichi, Japan), but failed because the occluded branch was sharply curved at the end of the M1 segment. Next, we tried SAT. We navigated the Marksman to one of the visible M2 branches over a microguidewire (Fig. 2B). Then, 5-MAX ACE 60 (Penumbra, Alameda, CA, USA) was advanced coaxially to the patent branch (Fig. 2C). With this technique, a complete recanalization was achieved (Fig. 2D). Puncture to reperfusion time was 51 minutes. No evident intracranial hemorrhage was observed upon head CT just after thrombectomy.

\section{Case 2}

An 86-year-old man presented with sudden onset of consciousness disturbance and was presented to our hospital. MRI showed a high-intensity area in the left thalamus on 

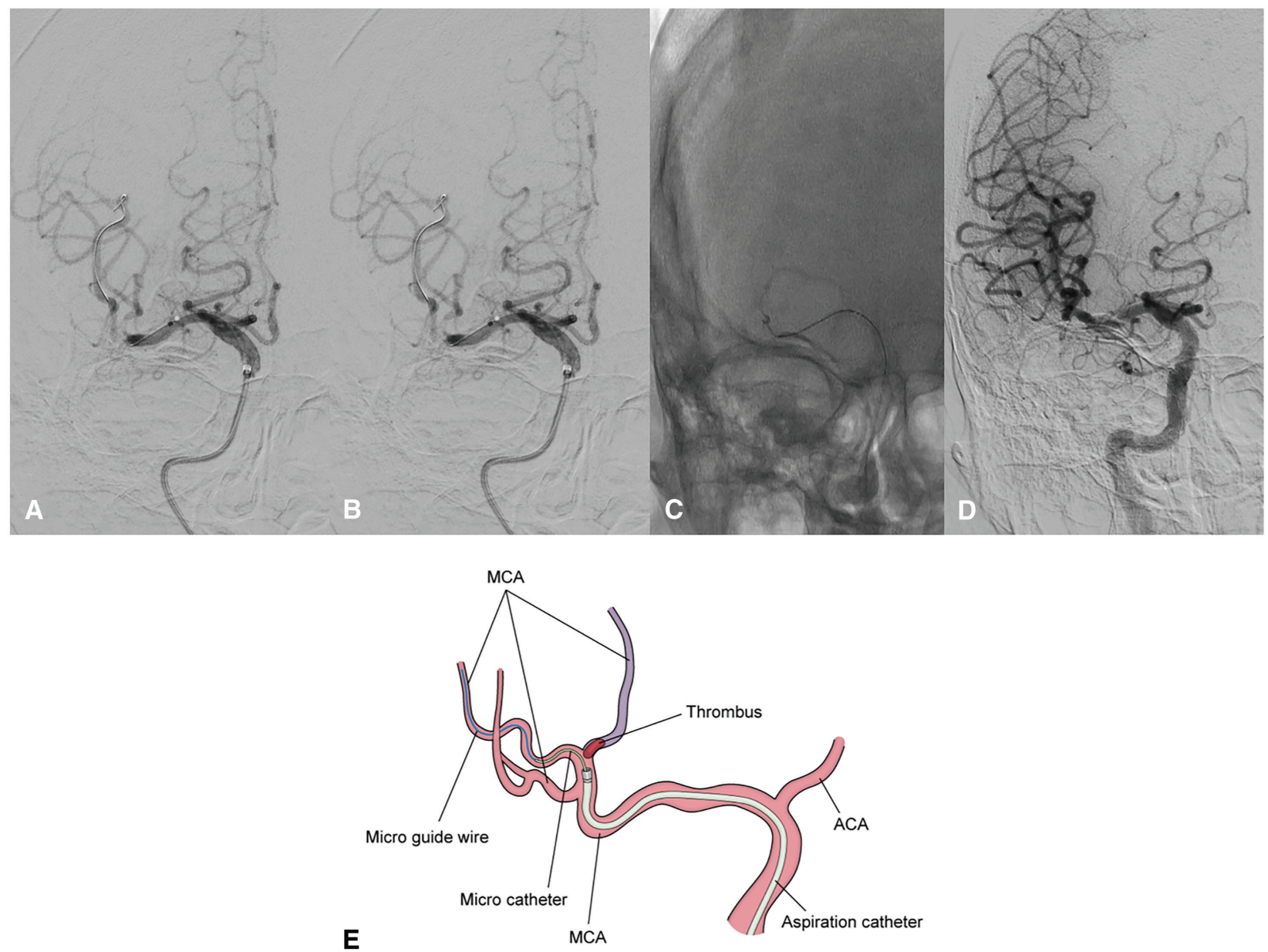

Fig. 2 Thrombectomy with the SAT for patient with MCA occlusion. (A) Pretreatment angiogram (anterior projection) showing occlusion of one of the M2 branches. (B) A microcatheter and a microguidewire are deployed into one of the visible M2 branches (C) and the aspiration catheter is deployed coaxially. (D) Repressing the reverse blood flow in the pump, aspiration catheter is carefully pulled back until the thrombus is caught. Posttreatment angiogram showed complete recanalization of the occluded left PCA. (E) An illustration of the right MCA and its branches in case 1. ACA: anterior cerebral artery; MCA: middle cerebral artery; PCA: posterior cerebral artery; SAT: sideway aspiration technique

DWI. Occlusion of the basilar artery was suspected on MRA.

The endovascular procedure was performed under conscious sedation. A 6 Fr ASAHI FUBUKI Dilator Kit (Asahi Intecc) was advanced into the basilar artery. Angiography revealed occlusion at the $\mathrm{P} 1$ segment of the left posterior cerebral artery (PCA), while the right PCA and its branches were barely patent (Fig. 3A). First, we tried to catheterize into the occluded left PCA but failed owing to the vessel tortuosity. Then, we introduced a velocity microcatheter (Penumbra) into the visible branch of the right PCA over an ASAHI CHIKAI black 14 soft tip (Asahi Intecc). We navigated a 4-MAX Penumbra catheter (Penumbra) coaxially with the velocity microcatheter and a microguidewire and attempted the SAT (Fig. 3B and $\mathbf{3 C}$ ). A red thrombus was retrieved and complete recanalization of thrombolysis in cerebral infarction grade 3 was achieved (Fig. 3D). Puncture to reperfusion time was 32 minutes. Head CT done immediately after the thrombectomy showed no evident intracranial hemorrhage.

\section{Discussion}

Standard techniques, namely stent retrieval and contact aspiration, can achieve good recanalization but do not work in some cases with tortured branches (or thrombus with some specific features). ${ }^{4-7)}$ We should be careful during thrombectomy for the distal vessel because of a high rate of perforation and distal embolization. ${ }^{8)}$ Mokin et al. reported an intraprocedural vessel perforation rate of $1.0 \%$ $(16 / 1599)$ in their case series, and half of them occurred at the M2/3 portion. ${ }^{9)}$ Furthermore, in case of posterior 

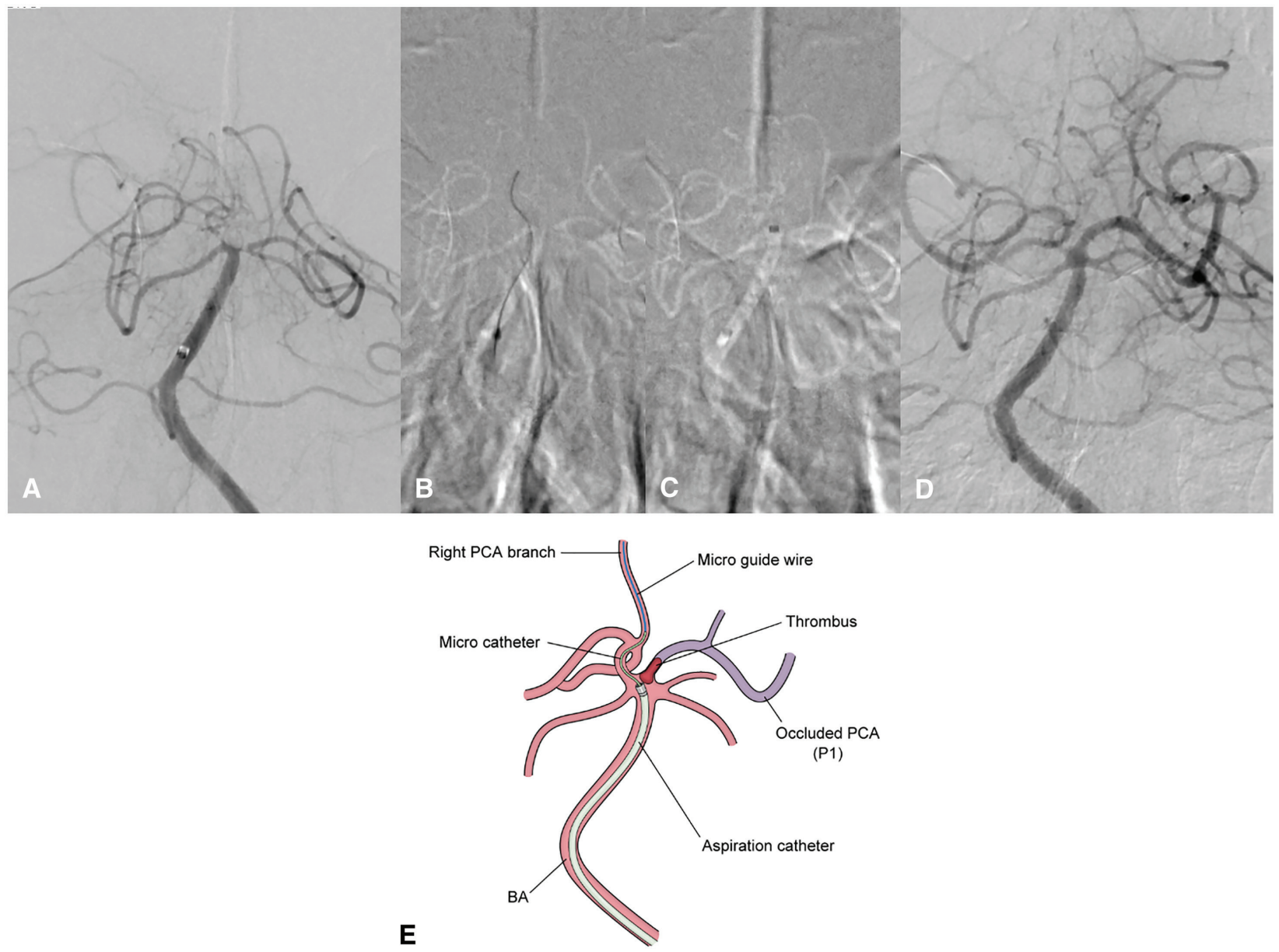

Fig. 3 Thrombectomy with the SAT for patient with the basilar tip occlusion. (A) Pretreatment angiogram (anterior projection) showing occlusion at the P1 of the left PCA, while the right PCA is barely patent. (B) A microguidewire and a microcatheter are deployed to the patent branch of right PCA. (C) Then, an aspiration catheter is advanced coaxially. The aspiration catheter is carefully pulled back repressing the reverse blood flow in the pump until it catches the thrombus. (D) Angiogram after thrombectomy using SAT showing complete recanalization of the occluded left PCA. (E) An illustration of the basilar artery system and catheter in case 2. BA: basilar artery; PCA: posterior cerebral artery; SAT: sideway aspiration technique

circulation occlusion, as in our case 2, de Leciñana et al. showed that arterial perforation during basilar artery occlusion occurred in $6.0 \%(3 / 52) .{ }^{10)}$

Agid and Power presented a modified method of stent retrieving called the tip of iceberg technique to overcome such complications. ${ }^{11)}$ In their two cases of successful recanalization for M2 occlusion, they deployed a stent retriever into a patent parent artery and captured the proximal side of the thrombus and pulled it out without catheterizing into the occluded vessel. Our SAT is a modification of their technique applied for contact aspiration, and it enables us to secure the thrombus more easily by suppressing the reverse blood flow. We consider our SAT is safe because this technique does not require introducing a larger diameter aspiration catheter or stent device into the branch that has a tiny diameter or is bifurcated sharply from the main artery.
The SAT has some limitations. First, unless a thrombus is close enough to the bifurcation or its tip is protruded into the parent artery, the vacuum effect of SAT cannot be expected. Second, this technique cannot be applied when the patent and visible artery are also bifurcated too sharply. In such a case, we cannot turn the contact surface area of the aspiration catheter to the thrombus, which may result in aspiration failure.

Lastly, like other contact aspiration techniques, suspension of or a marked decrease in the reverse flow from the aspiration catheter may sometimes indicate the impingement of the catheter tip against the vessel wall. Therefore, if we have difficulty pulling out the aspiration catheter, we need to draw out the catheter alternatively, for instance, taking the catheter out without applying negative pressure to it. 


\section{Conclusion}

We presented a new and alternative method for patients with thrombus in a sharply bifurcated branch. It warrants further study for safety and effectiveness.

\section{Author Contributions}

$\mathrm{MH}$ contributed to writing of the manuscript; TU and TM were the treating neurointerventionalists, contributed in acquisition of images and helped to create a draft of the manuscript; HI helped to create a draft of the manuscript; HS critically revised the manuscript for important intellectual content; and $\mathrm{EH}, \mathrm{KI}$, and KM critically revised the manuscript for intellectual content. All authors read and approved the final content of this case report.

\section{Patient Consent}

All patients provided written informed consent for participation in the study and for publication of the case report.

\section{Disclosure Statement}

The authors declare that there is no conflict of interest.

\section{References}

1) Deshaies EM. Tri-axial system using the Solitaire-FR and Penumbra Aspiration Microcatheter for acute mechanical thrombectomy. J Clin Neurosci 2013; 20: 1303-1305.

2) Turk AS, Turner R, Spiotta A, et al. Comparison of endovascular treatment approaches for acute ischemic stroke: cost effectiveness, technical success, and clinical outcomes. J Neurointerv Surg 2015; 7: 666-670.

3) Ohya $Y$, Uwatoko $T$, Mizokami $T$, et al. Use of middle cerebral artery visualization with coronal computed tomography to access target artery in mechanical thrombectomy. J Stroke Cerebrovasc Dis 2020; 29: 104714.

4) Kaymaz ZO, Nikoubashman O, Brockmann MA, et al. Influence of carotid tortuosity on internal carotid artery access time in the treatment of acute ischemic stroke. Interv Neuroradiol 2017; 23: 583-588.

5) Sporns PB, Hanning U, Schwindt W, et al. Ischemic stroke: histological thrombus composition and pre-interventional CT attenuation are associated with intervention time and rate of secondary embolism. Cerebrovasc Dis 2017; 44: 344-350.

6) Kaesmacher J, Boeckh-Behrens T, Simon S, et al. Risk of thrombus fragmentation during endovascular stroke treatment. AJNR Am J Neuroradiol 2017; 38: 991-998.

7) Gunning GM, McArdle K, Mirza M, et al. Clot friction variation with fibrin content; implications for resistance to thrombectomy. J Neurointerv Surg 2018; 10: 34-38.

8) Menon BK, Hill MD, Davalos A, et al. Efficacy of endovascular thrombectomy in patients with M2 segment middle cerebral artery occlusions: meta-analysis of data from the HERMES Collaboration. J Neurointerv Surg 2019; 11: 1065-1069.

9) Mokin M, Fargen KM, Primiani CT, et al. Vessel perforation during stent retriever thrombectomy for acute ischemic stroke: technical details and clinical outcomes. J Neurointerv Surg 2017; 9: 922-928.

10) de Leciñana MAlonso, Kawiorski MM, Ximénez-Carrillo Á, et al. Mechanical thrombectomy for basilar artery thrombosis: a comparison of outcomes with anterior circulation occlusions. J Neurointerv Surg 2017; 9: 1173-1178.

11) Agid R, Power S. Alternative technique for clot retrieval: the "tip of the iceberg" technique. Interv Neuroradiol 2015; 21: 703-706. 